\title{
Economic Burden of Patients with Inadequate Response to Targeted Immunomodulators for Rheumatoid Arthritis
}

\author{
Vibeke Strand, MD; Namita Tundia, PhD; Yan Song, PhD; \\ Dendy Macaulay, PhD; and Mahesh Fuldeore, MBA, PhD
}

\begin{abstract}
BACKGROUND: Targeted immunomodulators (TIMs), including biologic disease-modifying antirheumatic drugs (DMARDs) and JAK/STAT inhibitors, are effective therapies for rheumatoid arthritis (RA), but some patients fail to respond or lose response over time. This study estimated the realworld prevalence of RA patients with inadequate responses to an initial TIM (nonresponders) in the United States and assessed their direct and indirect economic burden compared with treatment responders.
\end{abstract}

METHODS: Administrative claims data (January 1999-March 2014) from a large private-insurer database were used, which included work-loss data from a subset of reporting companies. Eligible patients (classified as responders and nonresponders) had $\geq 1$ claim for a TIM approved for the treatment of RA and $\geq 2$ RA diagnoses in the claims history, with continuous pharmaceutical and medical benefit eligibility for 6 months before (baseline) and 12 months after (study period) the date of the first TIM claim (index date). All-cause and RA-related health care resource use (HCRU) and costs, work loss, and indirect costs during the study period were compared for responders versus nonresponders. Multivariable regression was used to adjust for baseline covariates. Sensitivity analyses of HCRU and direct costs were conducted for patients with index dates before and after 2008 to account for different approval dates of TIMs.

RESULTS: Of 7,540 eligible patients with RA, 2,527 (34\%) were classified as responders, and 5,013 (66\%) were classified as nonresponders; 407 and 723 had work-loss data, respectively. After adjusting for baseline covariates, nonresponders had significantly higher HCRU, including inpatient admissions (incidence rate ratio [IRR] $=1.94)$, outpatient visits $(I R R=1.19)$, emergency department visits (IRR $=1.53$ ), and number of prescription fills (IRR=1.09; all, $P<0.001)$. Nonresponders also had significantly higher adjusted all-cause ( $\$ 12,868$ vs. $\$ 9,621$, respectively) and RA-related ( $\$ 5,740$ vs. $\$ 4,495$; both, $P<0.001$ ) medical costs compared with responders. In addition, nonresponders reported significantly more days of work lost compared with responders ( 22.1 vs. 16.7 days, respectively; IRR $=1.21$; $P=0.007)$ and higher indirect costs $(\$ 3,548$ vs. $\$ 2,890 ; P=0.002)$.

Sensitivity analyses of HCRU and direct costs by index date (before and after 2008) were consistent with the full sample.

CONCLUSIONS: A large portion of patients with RA had inadequate responses to their initial TIM therapy with significantly higher economic burden, including higher HCRU, medical costs, and indirect costs due to work loss, compared with TIM therapy responders.

J Manag Care Spec Pharm. 2018;24(4):344-52

Copyright $\odot 2018$, Academy of Managed Care Pharmacy. All rights reserved.

\section{What is already known about this subject}

Targeted immunomodulators (TIMs), including inhibitors of tumor necrosis factor-alpha, interleukin-6, B-T cell interactions, B cell depletion, and JAK/STAT interactions, are specifically targeted, highly effective therapies for rheumatoid arthritis (RA); however, some patients still fail to respond or lose response over time.

When RA is left untreated or remains unresponsive to therapy, inflammation and progressive joint destruction lead to physical impairment, difficulties in maintaining employment, and significant economic burden.

\section{What this study adds}

This study estimated the real-world prevalence of RA patients with inadequate responses to initial TIM therapy in the United States and compared their direct and indirect costs, health care resource utilization, and lost days of work between responders and nonresponders using a large administrative claims database. Approximately $66 \%$ of RA patients treated with a TIM had inadequate responses to initial treatment and, compared with responders, experienced significantly more inpatient admissions, outpatient visits, emergency department visits, and prescription fills.

Nonresponders also had significantly higher all-cause and RA-related medical costs, significantly more days of work lost, and higher indirect costs compared with responders.

$\mathrm{R}$ heumatoid arthritis (RA) is a chronic disease that can lead to impairment in physical function due to inflammation causing stretching of tendons and ligaments, erosion of cartilage and bone, and ultimately destruction of joints. ${ }^{1}$ When active, RA symptoms can include tender and swollen joints, pain, fatigue, loss of energy, lack of appetite, low-grade fever, muscle and joint aches, and stiffness. ${ }^{2}$ RA is estimated to affect $0.5 \%-1 \%$ of adults in the United States, or about 1.5 million. ${ }^{3,4}$ It is more common among women and people aged 40 to 70 years, representing a large proportion of the working population in the United States. ${ }^{5,6}$ If left untreated or unresponsive to therapy, inflammation and progressive joint destruction can lead to impairment in physical function and inability to perform daily tasks, creating difficulties in maintaining employment. ${ }^{1}$ 
RA incurs a significant economic burden to individual patients and society. ${ }^{3}$ The estimated annual direct health care costs for a patient with RA were $\$ 2,000$ more than those for an individual without RA, resulting in total incremental costs of $\$ 22.3$ billion (in 2008 U.S. dollars [USD]) among all RA patients in the United States. ${ }^{7}$ In 1997, per capita employer expenditures (including direct health care costs and indirect costs due to disability and absenteeism) for employees with RA who had any disability claims were reported to be approximately 3 times higher $(\$ 17,822$ vs. $\$ 6,131)$ than those without RA. ${ }^{8}$ RA also results in high health care resource use (HCRU) in the United States, estimated at more than 250,000 hospitalizations and 9 million physician visits annually. ${ }^{9}$

Conventional treatment of RA has centered on judicious use of corticosteroids and disease-modifying antirheumatic drugs (DMARDs) such as methotrexate and leflunomide. ${ }^{10}$ Since 1998, targeted immunomodulators (TIMs) that inhibit tumor necrosis factor-alpha (TNFi), interleukin-6, B-T cell interactions, B cell depletion, and JAK/STAT interactions (Jakinibs) have been approved by the U.S. Food and Drug Administration (FDA) for treatment of RA based on efficacy in randomized controlled trials (RCTs) after failure of conventional DMARDs. ${ }^{11-16}$ TNFis (e.g., adalimumab, certolizumab pegol, etanercept, golimumab, and infliximab) were the first biologic therapies to be approved for RA, and RCT data demonstrate that these therapies are effective at improving clinical, functional, and patient-reported outcomes and halting radiographic structural progression. ${ }^{17-19}$ Jakinibs (e.g., tofacitinib) have similar efficacy to biologics. ${ }^{20}$ However, RCT data also indicate that a substantial proportion of patients do not adequately respond to TIM therapies. Approximately 40\% of RA patients treated with the above therapies fail to achieve American College of Rheumatology (ACR) 20\% response, and an even higher proportion of patients lose response over time or experience adverse events. ${ }^{21,22}$ Therapeutic failures are not limited to TNFis; similar failure rates associated with other RA treatments (e.g., abatacept, tocilizumab, rituximab, and tofacitinib) have also been reported. ${ }^{23-26}$

Although previous research has examined the economic burden in the overall RA patient population, limited data exist describing the economic burden of RA patients who fail to respond to TIM treatments. ${ }^{7.8}$ To address this gap in the literature, the objectives of this study were to estimate the realworld prevalence of RA patients who fail or have inadequate responses to initial TIM therapy in the United States and to compare their direct and indirect costs, HCRU, and work loss versus TIM responders.

\section{Methods}

\section{Data Source}

Data were procured from the database of OptumHealth Care Solutions (January 1999-March 2014), an administrative claims database of approximately 18.5 million privately insured individuals covered by 84 self-insured Fortune 500 companies with locations in all census areas of the United States (the top 500 public companies in the United States in terms of gross revenue). Data include all claims for all beneficiaries (i.e., employees, spouses, dependents, and retirees) nationwide, as well as short- and long-term disability claims for employees from a subset of 44 companies. This is a retrospective analysis of deidentified claims data compliant with the Health Insurance Portability and Accountability Act; thus, no review by an institutional review board was required.

\section{Study Population}

Patients were eligible for inclusion if they met the following criteria: (a) had at least 1 claim for a TIM therapy approved for the treatment of RA (abatacept, tocilizumab, tofacitinib, rituximab, anakinra, and TNFis), with or without the concomitant use of methotrexate, and the date of the first claim for a TIM (index agent) was defined as the index date; (b) had at least 1 diagnosis code for RA (International Classification of Diseases, Ninth Revision, Clinical Modification [ICD-9-CM] code 714.0) in the 6 months before the index date and at least 2 diagnoses of RA at any time in the patient's claims history; and (c) had continuous pharmaceutical and medical benefit eligibility for the 6 months before (baseline period) and 12 months after the index date (study period). Patients were excluded if they had (a) more than 1 TIM prescribed on the index date; (b) inconsistent reimbursement methods for the index agent (e.g., if there is a medical claim for a subcutaneous or oral index agent or a pharmacy claim for an intravenous index agent); or (c) any diagnosis during the baseline period for another condition for which any TIMs considered in this study are indicated (including ankylosing spondylitis, Crohn's disease, juvenile idiopathic arthritis, plaque psoriasis, psoriatic arthritis, and ulcerative colitis).

Selected patients were categorized as nonresponders or responders using a validated claims-based algorithm for determining treatment effectiveness developed by Curtis et al. (2011). ${ }^{27}$ Specifically, patients in the sample were classified as responders if they met all 6 of the following criteria in the study period:

1. High adherence: (a) For self-administered subcutaneous or oral agents (abatacept, adalimumab, anakinra, certolizumab, etanercept, golimumab, tocilizumab, and tofacitinib), this was determined as a medication possession ratio of $\geq 80 \%$, and (b) for agents administered via infusions (abatacept, golimumab, infliximab, and tocilizumab), this was determined as a number of infusions $\geq 80 \%$ of that recommended in their FDA labels. ${ }^{28}$

2. No increase in biologic dose or frequency during the maintenance period: (a) Within patients' claims, the number of infusions was within $120 \%$ of the number of scheduled infusions recommended in their FDA label, and (b) within patients' 


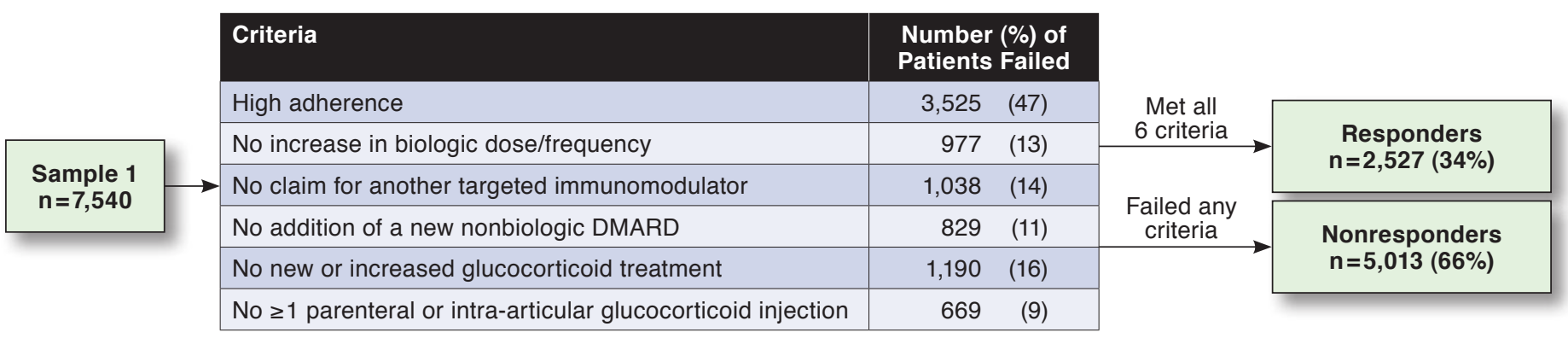

DMARD = disease-modifying antirheumatic drug; TIM = targeted immunomodulator .

claims, the weekly dose remained less than twice the standard weekly dose based on the FDA label.

3. No claim for another TIM approved for treatment of RA.

4. No claim for a new DMARD that the patient had not received at baseline.

5. No new or increased glucocorticoid treatment (in months 4-12 of the study period, no new oral glucocorticoid [ $\geq 30$-day supply] that the patient had not received at baseline; in months $7-12$, no increase $\geq 20 \%$ in dose of an existing oral glucocorticoid that the patient had received at baseline).

6. No parenteral or intra-articular glucocorticoid injection more than once over months 4-12 of the study period.

Criteria 1 and 2 did not apply to patients using rituximab because the FDA label does not provide a single recommended dosing schedule or glucocorticoid regimen for infusion. Patients who failed to meet any of the listed criteria were classified as nonresponders.

\section{Study Outcomes}

Baseline demographics (i.e., age, sex) were measured at the index date, and comorbidities and RA treatment history were measured during the baseline period. HCRU quantified during the study period included inpatient admissions and total length of stay, emergency department (ED) visits, outpatient visits, number of prescription fills, and other resource use (e.g., lab tests and services at home). All-cause direct health care costs (in 2014 USD) measured during the study period included pharmacy and medical service costs. Medical service costs excluding RA-related TIMs were separately estimated because responders likely received longer treatment by definition, and some agents were reimbursed in the medical claims. Thus, medical services costs excluding RA-related therapies may better reflect the actual burden in costs of medical services. RA-related costs were also estimated, including (a) costs in medical claims associated with a primary or secondary diagnosis of RA and (b) costs of RA-related therapies (TIMs, corticosteroids, nonbiologic DMARDs, and nonsteroidal anti-inflammatory drugs [NSAIDs]).

Days of disability and medically related work loss measured during the study period were based on data provided in disability claims. Medically related absenteeism was imputed based on use of medical services during business days (Monday through Friday) and gap days after the injury date and before the start of disability. It was assumed that each inpatient and ED visit accounted for a full day of work loss, while outpatient and other visits accounted for a half day of work loss. Indirect costs associated with disability and medically related absenteeism measured during the study period were based on actual short- and long-term disability payments paid by employers. Medically related absenteeism costs were calculated as the product of days of medically related absenteeism and the individual daily wage of each employee.

\section{Data Analysis}

For baseline characteristics, categorical characteristics were compared between responders and nonresponders using chisquare tests (Fisher's exact tests were used for outcomes with expected frequency $<5$ ). Continuous characteristics were compared between responders and nonresponders using Wilcoxon rank-sum tests.

For HCRU, days of disability, and medically related work loss, incidence rates (IRs) and overall proportions of events were estimated, and negative binomial and logistic regressions were used to obtain unadjusted and adjusted incidence rate ratios (IRRs) and odds ratios (ORs) comparing responders versus nonresponders. Adjusted covariates included age, sex, index year, Charlson Comorbidity Index (CCI), ${ }^{29}$ and individual baseline covariates with significant differences between the 2 cohorts. For direct and indirect health care cost outcomes, unadjusted costs were compared between cohorts using Wilcoxon rank-sum tests. Adjusted costs were estimated using a generalized linear model with a gamma distribution. For outcomes with $\geq 5 \%$ zeroes, a Tweedie distribution with an 


\begin{tabular}{|c|c|c|c|}
\hline \multicolumn{4}{|c|}{$\begin{array}{l}\text { TABLE 1 Baseline Characteristics of Responders } \\
\text { and Nonresponders to TIMs for RA }\end{array}$} \\
\hline Characteristics & $\begin{array}{c}\text { Responders } \\
\mathbf{n}=2,527\end{array}$ & $\begin{array}{c}\text { Nonresponders } \\
\mathbf{n}=5,013\end{array}$ & $P$ Value \\
\hline Age, mean (SD) & $56.9(12.7)$ & $56.1(13.2)$ & $0.005^{\mathrm{a}}$ \\
\hline Male, $\mathrm{n}(\%)$ & $730(28.9)$ & $1,151 \quad(23.0)$ & $<0.001^{\mathrm{a}}$ \\
\hline \multicolumn{4}{|l|}{ Year of index date, $\mathrm{n}(\%)$} \\
\hline $1998-2001$ & $219 \quad(8.7)$ & $390 \quad(7.8)$ & 0.182 \\
\hline $2002-2005$ & $824(32.6)$ & $1,511(30.1)$ & $0.029^{a}$ \\
\hline $2006-2009$ & $806 \quad(31.9)$ & $1,494 \quad(29.8)$ & 0.062 \\
\hline $2010-2013$ & $678(26.8)$ & $1,618 \quad(32.3)$ & $<0.001^{\mathrm{a}}$ \\
\hline \multicolumn{4}{|l|}{ Comorbidities } \\
\hline CCI, mean (SD) & $1.4 \quad(0.9)$ & $(1.0)$ & $0.002^{\mathrm{a}}$ \\
\hline \multicolumn{4}{|c|}{ Selected comorbidities, n (\%) } \\
\hline Osteoarthritis & $500 \quad(19.8)$ & 1,197 (23.9) & $<0.001^{\mathrm{a}}$ \\
\hline Gastrointestinal disease & $367(14.5)$ & $1,010(20.1)$ & $<0.001^{\mathrm{a}}$ \\
\hline Osteoporosis & $177 \quad(7.0)$ & $471 \quad(9.4)$ & $0.001^{\mathrm{a}}$ \\
\hline Enteropathic arthritis & $(0.3)$ & $(0.1)$ & 0.143 \\
\hline Reactive arthritis & $3(0.1)$ & $(0.3)$ & 0.165 \\
\hline \multicolumn{4}{|c|}{ Extra-articular manifestation of RA } \\
\hline Sjögren's syndrome & $38 \quad(1.5)$ & $101 \quad(2.0)$ & 0.120 \\
\hline Rheumatoid nodules & $20 \quad(0.8)$ & $(0.6)$ & 0.387 \\
\hline Rheumatoid lung & $(0.4)$ & $(0.5)$ & 0.886 \\
\hline Felty's syndrome & $5 \quad(0.2)$ & $(0.2)$ & 0.720 \\
\hline \multicolumn{4}{|c|}{ Treatment received during baseline period, $\mathbf{n}(\%)$} \\
\hline Glucocorticoid & $1,568(62.0)$ & $3,373(67.3)$ & $<0.001^{\mathrm{a}}$ \\
\hline Pain medication & $1,776(70.3)$ & $3,537(70.6)$ & 0.804 \\
\hline Prescription NSAIDs & $1,369(54.2)$ & $2,486 \quad(49.6)$ & $<0.001^{\mathrm{a}}$ \\
\hline Narcotic analgesics & $932(36.9)$ & $2,408(48.0)$ & $<0.001^{\mathrm{a}}$ \\
\hline Non-narcotic analgesics & $51 \quad(2.0)$ & $136 \quad(2.7)$ & 0.067 \\
\hline Nonbiologic DMARDs & $2,125 \quad(84.1)$ & $3,942 \quad(78.6)$ & $<0.001^{\mathrm{a}}$ \\
\hline Methotrexate & $1,733(68.6)$ & $3,076 \quad(61.4)$ & $<0.001^{\mathrm{a}}$ \\
\hline Hydroxychloroquine & $605(23.9)$ & $1,079 \quad(21.5)$ & $0.017^{\mathrm{a}}$ \\
\hline Leflunomide & $348 \quad(13.8)$ & $761 \quad(15.2)$ & 0.103 \\
\hline Sulfasalazine & $233 \quad(9.2)$ & $403 \quad(8.0)$ & 0.082 \\
\hline Minocycline & $(1.2)$ & $(1.4)$ & 0.501 \\
\hline Gold compound & $(0.3)$ & $(0.2)$ & 0.427 \\
\hline Mycophenolate & $(0.2)$ & $(0.3)$ & 0.160 \\
\hline Other & $(0.3)$ & $14 \quad(0.3)$ & 0.777 \\
\hline \multicolumn{4}{|c|}{$\begin{array}{l}\text { aP<0.05. } \\
C C I=\text { Charlson Comorbidity Index; DMARD = disease-modifying antirheumatic } \\
\text { drug; NSAID = nonsteroidal anti-inflammatory drug; RA=rheumatoid arthritis; } \\
S D=\text { standard deviation; } T I M=\text { targeted immunomodulator. }\end{array}$} \\
\hline
\end{tabular}

exponent of 1.67 was used. Adjusted covariates included age, sex, index year, CCI, and individual baseline covariates with significant differences between the 2 cohorts.

Because different TIMs were approved over the period for the treatment of RA (etanercept, infliximab, adalimumab, abatacept, rituximab, and anakinra were approved before 2008; tocilizumab, golimumab, tofacitinib, and certolizumab pegol were approved after 2008), the patterns of HCRU and direct health care costs might vary across different periods. Thus, as a sensitivity analysis, the analyses of HCRU and direct costs were further stratified by index year (before and after 2008).

\section{Results}

Of 7,540 eligible RA patients identified, 2,527 were categorized as responders and 5,013 as nonresponders (Figure 1). The most common index agent was etanercept (43\% of all patients), followed by adalimumab (26\%) and infliximab (22\%). Others included abatacept, rituximab, golimumab, anakinra, certolizumab pegol, tocilizumab, and tofacitinib (all $<3 \%$ ). Of the 6 criteria used to classify TIM responders and nonresponders, the criteria with the highest failure rates were "high adherence" (47\% of patients), "no new or increased glucocorticoid treatment" (16\%), and "no claim for another biologic" (14\%). Among all patients, 1,130 (407 responders and 723 nonresponders) were identified as the primary policy holder and continuously employed at a company that provided work-loss data during the entire study period, making them available for use in the work-loss and indirect-cost analyses.

Nonresponders were slightly younger (56.9 vs. 56.1 years, respectively; $P=0.005)$ and more likely to be female $(71.1 \%$ vs. $77.0 \% ; P<0.001$; Table 1). They had a significantly higher CCI than responders (mean score of 1.5 vs. 1.4, respectively; $P=0.002$ ) and were more likely to have osteoarthritis (23.9\% vs. $19.8 \%$ ), gastrointestinal disease (20.1\% vs. $14.5 \%)$, and osteoporosis (9.4\% vs. $7.0 \%$; all $P<0.001$ ). Additionally, nonresponders were significantly more likely to have taken glucocorticoids (67.3\% vs. $62.0 \%$, respectively) and narcotic analgesics (48.0\% vs. $36.9 \%$; both, $P<0.001)$ than responders but less likely to have received prescription NSAIDs (49.6\% vs. 54.2\%; $P<0.001)$, methotrexate (61.4\% vs. $68.6 \% ; P<0.001)$, or hydroxychloroquine $(21.5 \%$ vs. $23.9 \% ; P=0.017)$. There were slight differences between patients with index dates before versus after 2008: those with index dates after 2008 had a higher prevalence of osteoarthritis but a lower prevalence of osteoporosis or stroke. There was also lower use of pain medications (primarily prescription NSAIDs) among patients with index dates after 2008.

Nonresponders had significantly higher HCRU across all resource categories examined (Table 2): more inpatient admissions ( 0.4 vs. 0.2 events, respectively; IRR $=1.94 ; 95 \%$ confidence interval $[\mathrm{CI}]=1.70-2.21)$, days of hospitalization $(2.3$ vs. 0.8 days; IRR $=2.46 ; 95 \% \mathrm{CI}=2.0-2.93$ ), outpatient visits $(\mathrm{IRR}=1.19 ; 95 \% \mathrm{CI}=1.16-1.23)$, ED visits (IRR=1.53; 95\% CI $=1.38-1.69$ ), and number of total prescription fills (IRR $=1.09 ; 95 \% \mathrm{CI}=1.06-1.13)$ after adjustment for baseline covariates. Furthermore, the odds of any inpatient admission $(\mathrm{OR}=1.89 ; 95 \% \mathrm{CI}=1.65-2.17)$, $\mathrm{ED}$ visit $(\mathrm{OR}=1.60 ; 95 \%$ $\mathrm{CI}=1.43-1.79)$, or other visit $(\mathrm{OR}=1.17 ; 95 \% \mathrm{CI}=1.06-1.30)$ were all higher for nonresponders than responders after adjustment for baseline characteristics. Consistent with the full sample, for patients with an index year either before or after 2008, nonresponders had significantly higher HCRU across all categories: inpatient admissions, outpatient visits, ED visits, other visits, and number of prescription fills (all $P<0.05$ ). 
TABLE 2 Comparison of Health Care Resource Utilization and Work Loss Between Responders and Nonresponders to TIMs for RA

\begin{tabular}{|c|c|c|c|c|}
\hline & Responders & Nonresponders & Adjusted IRR (95\% CI) & $P$ Value \\
\hline Health care resource utilization & $\mathrm{n}=2,527$ & $\mathrm{n}=5,013$ & & \\
\hline \multicolumn{5}{|l|}{ Number of events per patient per year, IRR } \\
\hline Inpatient admissions & 0.2 & 0.4 & $(1.70-2.21)$ & $<0.001^{\mathrm{a}}$ \\
\hline Days of hospitalization & 0.8 & 2.3 & $2.46 \quad(2.06-2.93)$ & $<0.001^{\mathrm{a}}$ \\
\hline Outpatient visits & 18.8 & 23.6 & $1.19 \quad(1.16-1.23)$ & $<0.001^{\mathrm{a}}$ \\
\hline ED visits & 0.4 & 0.7 & $(1.38-1.69)$ & $<0.001^{\mathrm{a}}$ \\
\hline Other visits & 3.2 & 3.9 & $(1.05-1.23)$ & $0.001^{\mathrm{a}}$ \\
\hline Prescription fills & 42.5 & 49.0 & $(1.06-1.13)$ & $<0.001^{\mathrm{a}}$ \\
\hline \multicolumn{5}{|l|}{ Any events (\% of patients), OR } \\
\hline Any inpatient admission & 13.6 & 23.7 & $(1.65-2.17)$ & $<0.001^{\mathrm{a}}$ \\
\hline Any ED visit & 23.6 & 34.8 & $(1.43-1.79)$ & $<0.001^{\mathrm{a}}$ \\
\hline Any other visit & 56.5 & 61.7 & $(1.06-1.30)$ & $0.002^{\mathrm{a}}$ \\
\hline Work loss & $\mathrm{n}=407$ & $\mathrm{n}=723$ & & \\
\hline \multicolumn{5}{|l|}{ Days of work loss, IRR } \\
\hline Total days of work loss & 16.7 & 22.1 & $1.21 \quad(1.05-1.39)$ & $0.007^{\mathrm{a}}$ \\
\hline Days of disability & 7.2 & 10.6 & $(0.18-5.08)$ & 0.953 \\
\hline Days of medically related absenteeism & 9.5 & 11.5 & $1.14 \quad(1.04-1.24)$ & $0.006^{\mathrm{a}}$ \\
\hline \multicolumn{5}{|l|}{ Any work loss (\% of patients), OR } \\
\hline Any disability & 6.1 & 8.4 & $1.17 \quad(0.71-1.95)$ & 0.536 \\
\hline
\end{tabular}

In addition to higher HCRU, nonresponders also had significantly higher all-cause $(\$ 12,868$ vs. $\$ 9,621$, respectively) and RA-related ( $\$ 5,740$ vs. $\$ 4,495$; both $P<0.001$; Figure 2) medical costs after adjustment for baseline characteristics. Excluding costs of RA-related TIMs that were noted to be reimbursed in medical claims, adjusted all-cause inpatient $(\$ 2,789$ vs. $\$ 1,305$, respectively), outpatient ( $\$ 4,861$ vs. $\$ 3,798)$, ED (\$393 vs. $\$ 285$ ), and other ( $\$ 402$ vs. $\$ 312$; all, $P<0.05$ ) costs were all higher for nonresponders. A similar finding was evident for RA-related medical costs, although differences between the cohorts' inpatient and ED costs were not statistically significant (Figure 2). In the sensitivity analysis, considering patients with index years either before or after 2008, all-cause and RA-related total medical costs were higher for nonresponders (all, $P<0.001$ ). All 4 components of all-cause medical costs (inpatient, outpatient, ED, and other) were higher for nonresponders in the sensitivity analysis, although differences in ED and other costs were not statistically significant in patients with index years before 2008 .

Comparing the cohorts' work loss, nonresponders had significantly more days of work loss than responders (22.1 vs. 16.7, respectively; $I R R=1.21 ; 95 \% C I=1.05-1.39$ ), primarily driven by days of medically related absenteeism (11.5 vs. 9.5 days; IRR $=1.14 ; 95 \% \mathrm{CI}=1.04-1.24$; Table 2). The number of days of disability was numerically higher for nonresponders (10.6 vs. 7.2 days); however, adjusted IRR comparing the 2 groups was not statistically significant. In addition, the percentage of patients in each cohort with any work loss was similar (responders $=6.1 \%$, nonresponders $=8.4 \%$; IRR $=1.17$; 95\% CI=0.71-1.95). After adjustment for baseline covariates, nonresponders had significantly higher indirect costs ( $\$ 3,548$ vs. $\$ 2,890$, respectively; $P=0.002$ ), driven primarily by higher medically related absenteeism costs ( $\$ 2,323$ vs. $\$ 2,071$; $P=0.047$; Figure 3). Disability costs were numerically higher among nonresponders but did not differ significantly between the 2 cohorts ( $\$ 816$ vs. $\$ 575 ; P=0.458$ ).

\section{Discussion}

This retrospective claims study found that among RA patients treated with TIM therapy, approximately two thirds had inadequate responses to the first TIM they initiated. Treatment nonresponses were associated with increased HCRU and higher all-cause (difference of $\$ 3,247$ per patient) and RA-related (difference of $\$ 1,247$ per patient) medical costs during the study period. In addition, nonresponders experienced significantly higher indirect costs by $\$ 658$ due to higher costs of medically related absenteeism and more days of work lost. These results were consistent across 2 different time periods encompassing different FDA approval dates: patients who initiated therapies during 1998-2007 or 2008-2013 had similar outcomes to the full sample.

While TIMs significantly improved outcomes in RA patients compared with conventional synthetic DMARDs, a substantial proportion of patients fail to respond to these therapies. 


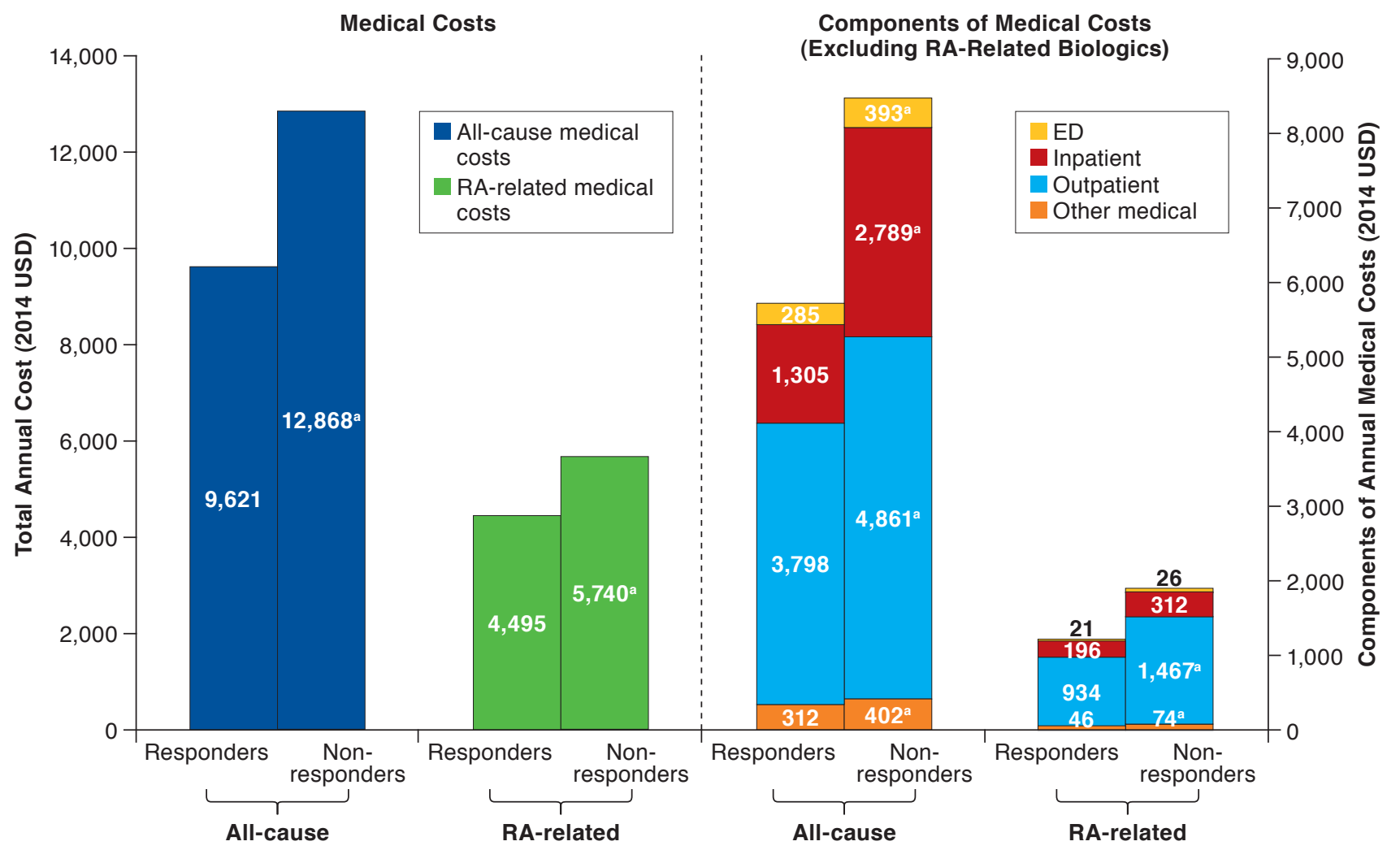

a $P<0.05$ : all-cause ED costs, $P=0.001$; all-cause other costs, $P=0.041$; RA-related other costs, $P=0.002$; all others, $P<0.001$.

$E D=$ emergency department; $R A=$ rheumatoid arthritis; TIM=targeted immunomodulator; USD = United States dollars.

For example, approximately 30\%-40\% of RA patients treated with a TNFi fail to achieve an ACR 20\% response, and more lose responses over time or experience adverse events. ${ }^{21}$ Nonresponses lead to treatment discontinuations or switching, and a recent claims database study of newly initiated TNFi continuers, discontinuers, and switchers noted that approximately one third of patients initiating a TNFi either switched therapies or discontinued within the first year and 50\% within 2 years. ${ }^{30,31}$ Long-term TIM discontinuation rates are even higher among RA patients. A study examining self-reported discontinuation during 1998-2011 found that 48\% discontinued TNFi or switched therapies; this rate was lower than the discontinuation rate of non-TNFi therapies (for first biologic, hazard ratio $=0.49$ [95\% CI $=0.34-0.71])^{32}$ Until recently, treatment options were limited for patients who were not satisfactorily responding to TIM therapies. Patients with RA who discontinue a TIM therapy are still likely to exhibit a significant response to a subsequent agent within the same category (e.g., TNFi). ${ }^{33}$ However, research in clinical practice settings has suggested that the best option for managing TIM nonresponders may be switching the patient to a drug with a different mechanism of action instead of to a different TNFi. ${ }^{17,34,35}$ For example, the 2015 ACR guidelines now conditionally recommend switching to a non-TNFi agent after initial TNFi failure. ${ }^{36}$ Similarly, it is possible that switching to a TIM agent with a mechanism of action unrelated to the initial treatment may also be a more effective option for TIM agents other than TNFis.

A better understanding of the reasons for a patient's inadequate response to an initial TIM may improve clinical decision making by helping to predict the response and tolerance to a subsequent TIM. However, the mechanism of suboptimal therapeutic response to TIMs for some patients with RA is still not well understood. New potential treatment options for this challenging patient population are under clinical investigation. For example, in a phase IIb clinical trial, upadacitinib (ABT-494), a selective JAKl inhibitor, added to methotrexate showed rapid improvement in RA signs and symptoms among patients with inadequate response or intolerance to a TNFi. ${ }^{37}$ 


\section{FIGURE 3 Comparison of Indirect Costs Between Responders and Nonresponders to TIMs for RA}

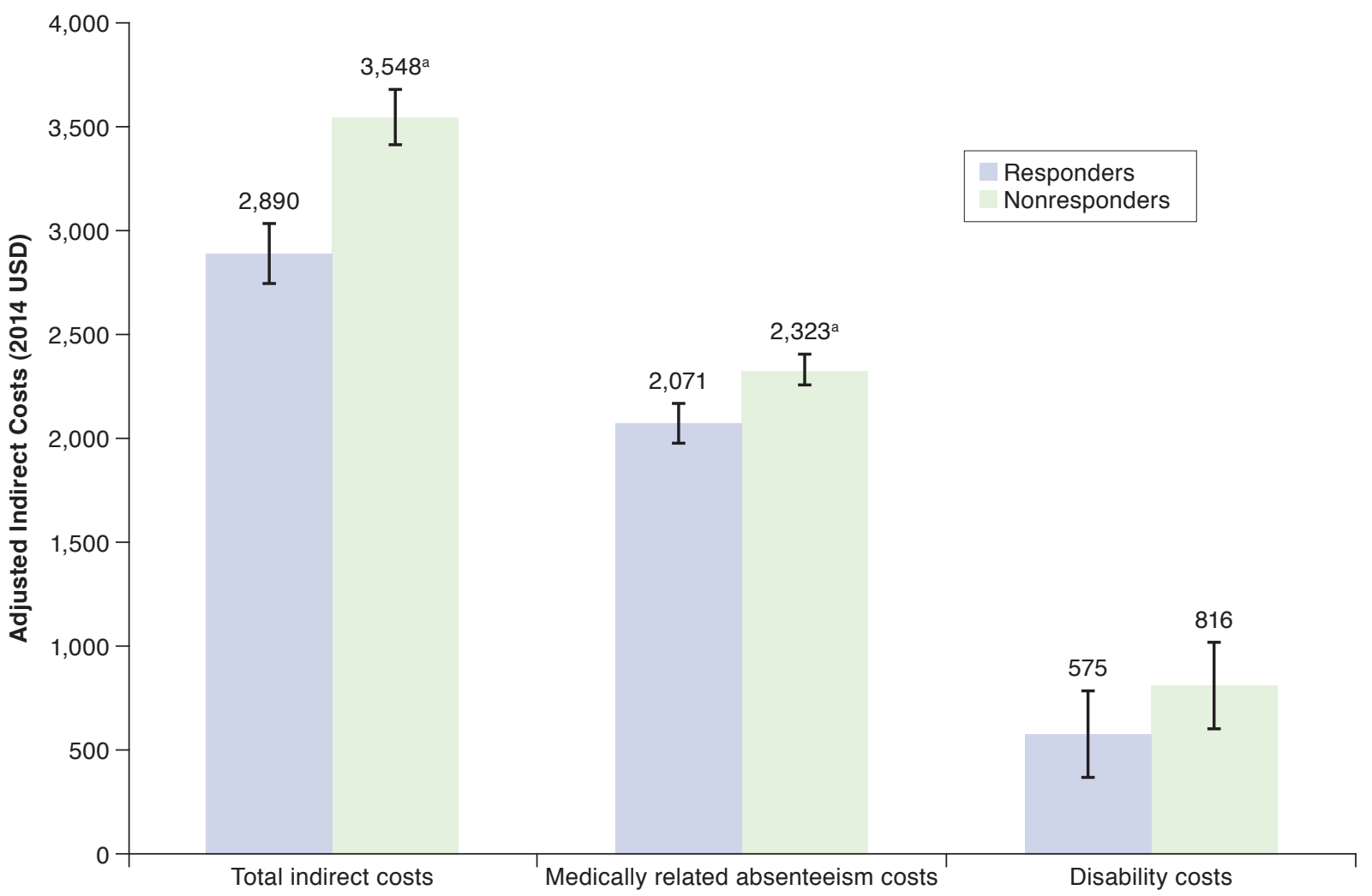

aP<0.05: total indirect costs, $P=0.002$; medically related absenteeism costs, $P=0.047$.

RA = rheumatoid arthritis; TIM = targeted immunomodulator; USD =U.S. dollars.

The findings of this study should be interpreted in light of several limitations. First, the data used in this analysis were limited to patients in commercial insurance plans; therefore, findings may not be generalizable to populations beyond the commercial plans. In particular, patients without insurance coverage or with public plans (e.g., Medicaid or Veterans Administration plans) may be likely to experience even greater burdens. Moreover, for patients over 65 years old, who could be partially covered by Medicare, the data capture the portion covered by private insurers but not the portion covered by Medicare; thus, HCRU and costs may be underestimated for these patients. Second, response to TIM treatment was evaluated using an algorithm based on administrative claims data, which may lead to misclassification. However, previous studies have validated the algorithm against a clinical evaluation of response (sensitivity $=0.75$, specificity $=0.90$ ). ${ }^{27}$ Third, confounding factors beyond those measured at baseline, such as the severity of RA or type of job, were not available and were not controlled for. Finally, this study is subject to the limitations of retrospective studies based on health care claims data.
These limitations may include (a) possible coding errors or omissions of claims, (b) multiple identities associated with the same individual if the individual changed employer and both employers contribute data to the database, and (c) unavailability for data outside the period of data coverage. One potential impact to this study is that, although patients were identified as having initiated TIM therapy, it is possible that they had used a TIM agent prior to the 6-month baseline period if that use occurred outside the coverage of the database. For example, it could be at a job with a different employer not included in the database or prior to the date that the employer started contributing data to the database. However, the current sample selection criteria ensured that the patient did not have any prescription of TIM for a period of at least 6 months prior to the index date. Therefore, the index drug captured in this study can be the first initiation of the drug or reinitiation after a discontinuation period of at least 6 months. These claims data-related limitations likely affect both the responder and nonresponder cohorts similarly and are therefore unlikely to bias the main findings. 


\section{Conclusions}

A considerable proportion of RA patients had inadequate responses to the first TIM they initiated, which was associated with increased HCRU, medical costs, and indirect costs due to medically related absenteeism. This high burden underscores the need for future research on mechanisms of nonresponses in RA, the development of effective therapies with lower rates of failure, and expansion of treatment approaches to address nonresponders, such as switching between TIMs in appropriately selected patients.

\section{Authors}

VIBEKE STRAND, MD, Division of Immunology/Rheumatology, Stanford University School of Medicine, Palo Alto, California. NAMITA TUNDIA, PhD, and MAHESH FULDEORE, MBA, PhD, AbbVie, North Chicago, Illinois. YAN SONG, PhD, and DENDY MACAULAY, PhD, Analysis Group, Boston, Massachusetts.

AUTHOR CORRESPONDENCE: Namita Tundia, PhD, 1 N. Waukegan Rd., D-GMH1, AP31-1 NE, North Chicago, IL 60064. Tel.: 847.938.9482; E-mail: namita.tundia@abbvie.com.

\section{DISCLOSURES}

Funding for this research was provided by AbbVie, which was involved in all stages of the study research and manuscript preparation. Tundia and Fuldeore are employed by AbbVie. Song and Macaulay are employed by Analysis Group, which received grants from AbbVie to conduct this study. Strand reports grants and personal fees from AbbVie, Amgen, AstraZeneca, Bayer, Boehringer Ingelheim, Celltrion, Corrona, Crescendo, Genentech/Roche, GSK, Janssen, Lilly, Novartis, Pfizer, Regeneron, Samsung, Sandoz, Sanofi, and UCB outside the submitted work.

Study concept and design were contributed by Tundia, Song, and Macaulay, along with other authors. Data analyses were designed and conducted by Song and Macaulay. All authors contributed to data interpretation. Writing of the manuscript was led by Tundia, Song, and Macaulay, with revisions by all authors.

A synopsis of the current research was presented at the American College of Rheumatology/Association of Rheumatology Health Professionals meeting, which took place in Washington, DC, during November 11-16, 2016.

\section{ACKNOWLEDGMENTS}

Medical writing assistance was provided by Shelley Batts, $\mathrm{PhD}$, an employee of Analysis Group. The authors thank Zhou Zhou, Cassie Tang, and Debbie Goldschmidt from Analysis Group for analytical support. Financial support for these services was provided by AbbVie.

\section{REFERENCES}

1. Venables P, Ravinder N. Diagnosis and differential diagnosis of rheumatoid arthritis. Available at: http://www.uptodate.com/contents/diagnosisand-differential-diagnosis-of-rheumatoid-arthritis. Accessed March 1, 2018.

2. Hootman JM, Helmick CG. Projections of U.S. prevalence of arthritis and associated activity limitations. Arthritis Rheum. 2006;54(1):226-29.
3. Kvien TK. Epidemiology and burden of illness of rheumatoid arthritis. PharmacoEconomics. 2004;22(2 Suppl 1):1-12.

4. Helmick CG, Felson DT, Lawrence RC, et al. Estimates of the prevalence of arthritis and other rheumatic conditions in the United States. Part I. Arthritis Rheum. 2008;58(1):15-25.

5. Myasoedova E, Crowson CS, Kremers HM, Therneau TM, Gabriel SE. Is the incidence of rheumatoid arthritis rising?: results from Olmsted County, Minnesota, 1955-2007. Arthritis Rheum. 2010;62(6):1576-82.

6. Lee DM, Weinblatt ME. Rheumatoid arthritis. Lancet. 2001;358(9285):903-11.

7. Kawatkar AA, Jacobsen SJ, Levy GD, Medhekar SS, Venkatasubramaniam KV, Herrinton LJ. Direct medical expenditure associated with rheumatoid arthritis in a nationally representative sample from the medical expenditure panel survey. Arthritis Care Res (Hoboken). 2012;64(11):1649-56.

8. Birnbaum HG, Barton M, Greenberg PE, et al. Direct and indirect costs of rheumatoid arthritis to an employer. J Occup Environ Med. 2000;42(6):588-96

9. American College of Rheumatology Subcommittee on Rheumatoid Arthritis Guidelines. Guidelines for the management of rheumatoid arthritis: 2002 update. Arthritis Rheum. 2002;46(2):328-46.

10. Fries JF. Current treatment paradigms in rheumatoid arthritis. Rheumatology (Oxford). 2000;39(Suppl 1):30-35.

11. Scott DL. Biologics-based therapy for the treatment of rheumatoid arthritis. Clin Pharmacol Ther. 2012;91(1):30-43.

12. Keystone EC, Kavanaugh AF, Sharp JT, et al. Radiographic, clinical, and functional outcomes of treatment with adalimumab (a human anti-tumor necrosis factor monoclonal antibody) in patients with active rheumatoid arthritis receiving concomitant methotrexate therapy: a randomized, placebocontrolled, 52-week trial. Arthritis Rheum. 2004;50(5):1400-11.

13. St Clair EW, van der Heijde DM, Smolen JS, et al. Combination of infliximab and methotrexate therapy for early rheumatoid arthritis: a randomized, controlled trial. Arthritis Rheum. 2004;50(11):3432-43.

14. Klareskog L, van der Heijde D, de Jager JP, et al. Therapeutic effect of the combination of etanercept and methotrexate compared with each treatment alone in patients with rheumatoid arthritis: double-blind randomised controlled trial. Lancet. 2004;363(9410):675-81.

15. Ruiz Garcia V, Jobanputra P, Burls A, et al. Certolizumab pegol (CDP870) for rheumatoid arthritis in adults. Cochrane Database Syst Rev. 2011(2):CD007649.

16. Keystone E, Genovese MC, Klareskog L, et al. Golimumab in patients with active rheumatoid arthritis despite methotrexate therapy: 52-week results of the GO-FORWARD study. Ann Rheum Dis. 2010;69(6):1129-35.

17. Emery P. Optimizing outcomes in patients with rheumatoid arthritis and an inadequate response to anti-TNF treatment. Rheumatology (Oxford). 2012;51(Suppl 5):v22-30.

18. Quinn MA, Conaghan PG, O'Connor PJ, et al. Very early treatment with infliximab in addition to methotrexate in early, poor-prognosis rheumatoid arthritis reduces magnetic resonance imaging evidence of synovitis and damage, with sustained benefit after infliximab withdrawal: results from a twelve-month randomized, double-blind, placebo-controlled trial. Arthritis Rheum. 2005;52(1):27-35.

19. van de Putte LB, Atkins C, Malaise M, et al. Efficacy and safety of adalimumab as monotherapy in patients with rheumatoid arthritis for whom previous disease modifying antirheumatic drug treatment has failed. Ann Rheum Dis. 2004;63(5):508-16.

20. Yamaoka K. Janus kinase inhibitors for rheumatoid arthritis. Curr Opin Chem Biol. 2016;32:29-33.

21. Rubbert-Roth A, Finckh A. Treatment options in patients with rheumatoid arthritis failing initial TNF inhibitor therapy: a critical review. Arthritis Res Ther. 2009;11(Suppl 1):S1. 
22. Karlsson JA, Kristensen LE, Kapetanovic MC, Gulfe A, Saxne T, Geborek P. Treatment response to a second or third TNF-inhibitor in RA: results from the South Swedish Arthritis Treatment Group Register. Rheumatology (Oxford). 2008;47(4):507-13

23. Kaneshita S, Min C, Fumio O, et al. Highly elevated rheumatoid factor is a risk factor for abatacept treatment failure in Japanese patients with rheumatoid arthritis. Abstract presented at: American College of Rheumatology; November 6-11, 2015; San Francisco, CA. Available at: http://acrabstracts. org/abstract/highly-elevated-rheumatoid-factor-is-a-risk-factor-for-abatacepttreatment-failure-in-japanese-patients-with-rheumatoid-arthritis/. Accessed March 1, 2018.

24. Navarro-Millan I, Singh JA, Curtis JR. Systematic review of tocilizumab for rheumatoid arthritis: a new biologic agent targeting the interleukin- 6 receptor. Clin Ther. 2012;34(4):788-802, e783.

25. Buch MH, Smolen JS, Betteridge N, et al. Updated consensus statement on the use of rituximab in patients with rheumatoid arthritis. Ann Rheum Dis. 2011;70(6):909-20.

26. Lee EB, Fleischmann R, Hall S, et al. Tofacitinib versus methotrexate in rheumatoid arthritis. New Engl J Med. 2014;370(25):2377-86.

27. Curtis JR, Baddley JW, Yang S, et al. Derivation and preliminary validation of an administrative claims-based algorithm for the effectiveness of medications for rheumatoid arthritis. Arthritis Res Ther. 2011;13(5):R155.

28. Sikka R, Xia F, Aubert RE. Estimating medication persistency using administrative claims data. Am J Manag Care. 2005;11(7):449-57.

29. Quan H, Sundararajan V, Halfon P, et al. Coding algorithms for defining comorbidities in ICD-9-CM and ICD-10 administrative data. Med Care. 2005;43(11):1130-39
30. Harnett J, Wiederkehr D, Gerber R, Gruben D, Koenig A, Bourret J Real-world evaluation of TNF-inhibitor utilization in rheumatoid arthritis. J Med Econ. 2016;19(2):91-102.

31. Strand V, Williams S, Miller PSJ, Saunders K, Grant S, Kremer JM. Discontinuation of biologic therapy in rheumatoid arthritis (RA): analysis from the Consortium of Rheumatology Researchers of North America (CORRONA) Database. Ann Rheum Dis. 2013;72:A71-A72.

32. Ramiro S, Landewe R, van der Heijde D, Harrison D, Collier D, Michaud $\mathrm{K}$. Discontinuation rates of biologics in patients with rheumatoid arthritis: are TNF inhibitors different from non-TNF inhibitors? RMD Open. 2015;1(1):e000155.

33. Lloyd S, Bujkiewicz S, Wailoo AJ, Sutton AJ, Scott D. The effectiveness of anti-TNF-alpha therapies when used sequentially in rheumatoid arthritis patients: a systematic review and meta-analysis. Rheumatology (Oxford). 2010;49(12):2313-21.

34. Favalli EG, Biggioggero M, Marchesoni A, Meroni PL. Survival on treatment with second-line biologic therapy: a cohort study comparing cycling and swap strategies. Rheumatology (Oxford). 2014;53(9):1664-68.

35. Hirabara S, Takahashi N, Fukaya N, et al. Clinical efficacy of abatacept, tocilizumab, and etanercept in Japanese rheumatoid arthritis patients with inadequate response to anti-TNF monoclonal antibodies. Clin Rheumatol. 2014;33(9):1247-54.

36. Singh JA, Saag KG, Bridges SL, Jr., et al. 2015 American College of Rheumatology guideline for the treatment of rheumatoid arthritis. Arthritis Rheumatol. 2016;68(1):1-26.

37. Kremer JM, Emery P, Camp HS, et al. A phase IIb study of ABT-494, a selective JAK-1 inhibitor, in patients with rheumatoid arthritis and an inadequate response to anti-tumor necrosis factor therapy. Arthritis Rheumatol. 2016;68(12):2867-77. 